\title{
Tarifpolitischer Jahresbericht 2009: Tarifverdienste mit kräftigem Plus - Effektivverdienste im Minus
}

Trotz der Wirtschaftskrise erzielten die Gewerkschaften im Jahr 2009 lohnpolitisch bemerkenswerte Erfolge. Die Tarifabschlüsse wiesen Steigerungsraten zwischen $2 \%$ und $4 \%$ auf. Die tariflichen Löhne und Gehälter stiegen damit im Durchschnitt real kräftig an. Anders das Bild bei den Effektiveinkommen: Die tatsächlich gezahlten Bruttolöhne und -gehälter sanken 2009. Zum sechsten Mal in Folge mussten die Beschäftigten eine Realeinkommenssenkung hinnehmen. Ursache dieser widersprüchlichen Entwicklung war vor allem die Kurzarbeit, aber auch Abstriche beim Entgelt, z. B. durch Nutzung tariflicher Öffnungsklauseln, spielten eine Rolle. Die Gewerkschaften zeigten sich im vergangenen Jahr - trotz der Krise - auch kampfbereit, wie die Tarifkonflikte im Gebäudereinigerhandwerk und beim Sozial- und Erziehungsdienst veranschaulichen.

\section{1 \\ Das Tarifjahr 2009 im Überblick}

\subsection{RAHMENBEDINGUNGEN}

Die Tarifpolitik stand 2009 unter dem Eindruck der Wirtschaftskrise. Der scharfe Einbruch bei Aufträgen, Produktion und Absatz, vor allem in den exportorientierten Industriebranchen, und die Sorge vor einer katastrophalen Entwicklung am Arbeitsmarkt bildeten sehr ungünstige Rahmenbedingungen insbesondere für die Lohnrunden des vergangenen Jahres. Zunächst lieferte jedoch der Abschluss für die Metallindustrie vom November 2008, der gewissermaßen in letzter Sekunde vor dem tiefen Absturz in diesem Wirtschaftsbereich erzielt werden konnte, mit einer Tariferhöhung von insgesamt 4,2\% eine positive Vorlage für die nachfolgenden Tarifrunden. Im öffentlichen Dienst, der naturgemäß von der Krise zunächst nicht direkt erfasst wurde, orientierte sich der Abschluss für die Länder Anfang März an dem ebenfalls positiven Ergebnis für den Bereich von Bund und Gemeinden aus dem Vorjahr. In der Folge konnten auch in anderen Branchen noch akzeptable Abschlüsse erzielt werden. Schließlich wirkten auch die länger laufenden Verträge aus dem Vorjahr mit kräftigen Stufenanhebungen für
2009 stabilisierend auf die Tarifentwicklung. Allerdings blieb die Tarifentwicklung von den Krisenfolgen nicht unberührt. So wurden in einer Reihe von Branchen Nullmonate und Pauschalzahlungen vereinbart, denen dann bescheidene Tarifanhebungen folgten. Im zweiten Halbjahr fielen die Abschlüsse insgesamt deutlich niedriger aus. Außerdem drängten die Arbeitgeberverbände vielfach mit Erfolg auf Krisenklauseln, die die betriebliche Umsetzung der Tarifergebnisse unter Vorbehalt stellten.

Auf betrieblicher Ebene dominierten insbesondere in den krisenbetroffenen Industriebranchen die Bemühungen um Beschäftigungssicherung. Während die prekären Beschäftigungsverhältnisse (Leiharbeit, befristete Beschäftigung) zumeist rasch abgebaut wurden, setzten Gewerkschaften und betriebliche Interessenvertretungen alles daran, betriebsbedingte Kündigungen zu vermeiden. „Keine Entlassungen in der Krise!" lautete eine zentrale Parole der IG Metall (2009). Dabei konnten die Gewerkschaften auch auf ein breit gefächertes tarifpolitisches Instrumentarium von befristeter Arbeitszeitverkürzung bis zur tariflich geregelten Kurzarbeit zurückgreifen (WSI-Tarifarchiv 2009a, 2009b). Insgesamt konnte der Anstieg der registrierten Arbeitslosigkeit in überraschend engen Grenzen gehalten werden, aber die durch Kurzarbeit und Abbau von Arbeitszeitkonten in Beschäftigung gehaltenen Arbeitnehme- rinnen und Arbeitnehmer erlitten entsprechende Einkommenseinbußen.

Das politische Klima zwischen den Tarifparteien blieb im Jahr 2009 insgesamt betrachtet relativ entspannt. Den 60 . Geburtstag des Tarifvertragsgesetzes im April nahmen beide Seiten zum Anlass, die Vorzüge der Tarifautonomie herauszustreichen (Sommer 2010; BDA 2009). Die Arbeitgeber gaben ausdrücklich zu Protokoll, dass sie nicht weiter auf einer Forderung nach gesetzlichen Öffnungsklauseln bestehen, und sie verwiesen in diesem Zusammenhang auf die zunehmende Flexibilität des Tarifsystems. Die Bundesvereinigung der Arbeitgeberverbände (BDA) zeigte sich auch mit den Ergebnissen der Tarifpolitik des vergangenen Jahres insgesamt sehr zufrieden. BDA-Präsident Dieter Hundt stellte auf dem Arbeitgebertag im November 2009 fest, die Tarifabschlüsse dieses Jahres seien ,angemessen, beschäftigungsorientiert, differenziert und flexibel gewesen " (Hundt 2009). Auch das Verhältnis der Ge-

Bispinck, Reinhard, Dr., Wissenschaftler im
WSI und Leiter des WSI-Tarifarchivs in der
Hans-Böckler-Stiftung.
e-mail: Reinhard-Bispinck@boeckler.de
Götz Bauer, Monika Müller, Ulrich Schmidt,
Monika Schwacke-Pilger, Andrea Taube und
Monika Wiebel sind Sachbearbeiterinnen
und Sachbearbeiter im WSI-Tarifarchiv.

Bispinck, Reinhard, Dr., Wissenschaftler im WSI und Leiter des WSI-Tarifarchivs in der Hans-Böckler-Stiftung. e-mail: Reinhard-Bispinck@boeckler.de Monika Schwacke-Pilger, Andrea Taube und und Sachbearbeiter im WSI-Tarifarchiv. 
werkschaften zur Politik war größtenteils kooperativ: Die alte schwarz-rote Bundesregierung hatte früh auf eine Politik der Beschäftigungssicherung (vor allem durch stark verlängerte Kurzarbeitsfristen) gesetzt, ihre schwarz-gelbe Nachfolgerin hielt daran zunächst fest. Auch bei der Rettung existenzbedrohter Unternehmen zeigte sich die Politik grundsätzlich kooperationsbereit, auch wenn es - wie das Beispiel Opel zeigt - Konflikte um die konkrete Ausgestaltung gab.

Konfliktbeladen blieb auch das Thema „Mindestlohn“: Die alte wie die neue Bundesregierung verweigerten sich der von den Gewerkschaften geforderten Einführung eines einheitlichen gesetzlichen Mindestlohnes. Und auch bei der Festlegung branchenbezogener Mindestlöhne nach dem Entsendegesetz wurden nur begrenzte Fortschritte erzielt. Die Arbeitgeber kritisierten ihrerseits scharf jegliche Versuche, Tarifverträge durch staatliche Lohnfestsetzung außer Kraft zu setzen.

\subsection{FORDERUNGEN UND ABSCHLÜSSE}

Auffällig war in der Tarifrunde 2009, dass die dramatische Krisenentwicklung zunächst kaum Spuren bei den gewerkschaftlichen Tarifforderungen hinterließ. Sie bewegten sich überwiegend auf hohem, teils sogar sehr hohem Niveau. Maßgebliche Begründung für die Tarifforderungen waren neben den Verteilungsansprüchen der Beschäftigten auch die gesamtwirtschaftlich notwendige Stabilisierung der Binnennachfrage, um einen Ausgleich für die wegbrechende Exportnachfrage zu schaffen. Hinzu kamen tarifbereichsspezifische Rahmenbedingungen: Im öffentlichen Dienst forderte die Vereinte Dienstleistungsgewerkschaft (ver.di) für die Beschäftigten der Länder eine Tarifanhebung von $8 \%$, mindestens jedoch $200 €$, und orientierte sich damit exakt an der Vorjahresforderung für den Bereich Bund und Gemeinden. Bei der Deutschen Telekom $(8,5 \%$, mindestens $220 €)$ und der Deutschen Bahn (10\%) argumentierten die Gewerkschaften mit den (bis dahin noch) guten Unternehmensbilanzen. Im Handel beliefen sich die Forderungen zwischen 6,5\% (Einzelhandel) und $8 \%$ (Großhandel). In den übrigen Bereichen bewegten sich die Forderungen überwiegend zwischen $5 \%$ und $6 \%$. In verschiedenen Branchen zielten die Gewerkschaften mit

Übersicht 1: Tarifforderungen in der Tarifrunde 2009 in ausgewählten Tarifbereichen

\begin{tabular}{llc}
\hline Tarifbereich & Gewerkschaft & Forderung \\
\hline Bauhauptgewerbe & IG BAU & $\mathbf{6 , 0} \%$ \\
Deutsche Bahn AG & Transnet & $\mathbf{1 0 , 0} \%$ \\
Deutsche Telekom AG & ver.di & $\mathbf{8 , 5} \%$, mind. $220 €$ \\
Druckindustrie & ver.di & $\mathbf{5 , 0} \%$ \\
Eisen- und Stahlindustrie & IG Metall & $\mathbf{4 , 5} \%$ \\
Elektrohandwerk NRW & IG Metall & $\mathbf{5 , 5} \%$ \\
Einzelhandel NRW & ver.di & $\mathbf{6 , 5} \%$, mind. $135 €$ \\
Energiewirtschaft Tarifgruppe RWE & IG BCE/ver.di & $\mathbf{8 , 0} \%$ \\
Gebäudereinigerhandwerk & IG BAU & $\mathbf{8 , 7} \%$ \\
Groß- und Außenhandel NRW & ver.di & $\mathbf{8 , 0} \%$, mind. $175 €$ \\
Holz- und Kunststoffindustrie & IG Metall & $\mathbf{5 , 5} \%$ \\
Maler- und Lackiererhandwerk & IG BAU & $\mathbf{7 , 2} \%$ \\
Nahrung-Genuss-Gaststätten & NGG & $\mathbf{5 , 0 - 7 , 0} \%$ \\
Öffentlicher Dienst (Länder) & ver.di & $\mathbf{8 , 0} \%$, mind. $200 €$ \\
Textil- und Bekleidungsindustrie West & IG Metall & $\mathbf{5 , 5} \%$ \\
Textilreinigungsgewerbe & IG Metall & $\mathbf{5 , 5} \%$ \\
\hline & & WSI MITTEILUNGEN \\
Quelle: WSI-Tarifarchiv. & &
\end{tabular}

Sockelbeträgen auf eine stärkere Tarifanhebung für die unteren Einkommensgruppen (Übersicht 1).

Nach dem Kündigungsterminkalender liefen die Tarifverträge im öffentlichen Dienst (Länder) Ende Dezember 2008 als Erste aus, zeitgleich auch in einigen Energiebereichen (RWE, Vattenfall) sowie bei der Deutschen Telekom. Ende Januar 2009 folgten die Deutsche Bahn, Ende Februar die Textil- und Bekleidungsindustrie (West) und Ende März das Bauhauptgewerbe, die Druckindustrie und die Eisen- und Stahlindustrie. Die verschiedenen regionalen Tarifbereiche des Einzelhandels sowie des Großhandels standen ebenfalls Ende März bzw. Ende April zu Verhandlungen an. Noch nicht beendet waren zu Beginn der Tarifrunde 2009 die Verhandlungen im Bankgewerbe, die im Oktober 2008 angesichts der Finanzkrise unterbrochen worden waren.

Eine wichtige Vorgabe für die Tarifrunde 2009 bildete - wie erwähnt - der Abschluss für die Metallindustrie. Im November 2008 hatten sich die Tarifparteien auf einen 18-monatigen Tarifabschluss geeinigt, der neben einer Pauschalzahlung von $510 €$ für die ersten drei Monate eine Tariferhöhung in zwei Stufen um jeweils 2,1 \% im Februar und Mai 2009 sowie eine weitere Sonderzahlung von $122 €$ im September 2009 vorsah.

Ebenfalls bereits im Jahr 2008 erfolgte ein Abschluss in der Energiewirtschaft. Er sah für die Tarifgruppe RWE eine Tarifanhebung von $4 \%$ ab Januar 2009, eine Stufenanhebung um 2,2 \% ab Beginn nächs- ten Jahres sowie zwei Einmalzahlungen von $1.200 €(2009)$ und $800 €(2010)$ mit einer Laufzeit bis 31.10.2010 vor. Vergleichbare Abschlüsse gab es in anderen Energiebereichen.

Ende Januar vereinbarten die Tarifparteien bei der Deutschen Bahn AG einen zweistufigen Abschluss mit einer Anhebung um 2,5\% ab 1.2.2009 und einer Stufenerhöhung um $2 \%$ ab 1.1.2010 sowie einer Einmalzahlung von $500 €$, Laufzeit bis 31.7.2010. Außerdem wurden verbesserte Arbeitszeitregelungen vereinbart. Bei der Deutschen Telekom AG sah der Abschluss eine Tarifsteigerung von $3 \%$ ab 1.1.2009 und weitere 2,5 \% ab 1.1.2010 vor. Für den Großteil der Beschäftigten im Bereich der Service-Gesellschaften der Deutschen Telekom AG erhöhten sich die Vergütungen zu den gleichen Zeitpunkten um $5 \%$ bzw. 3,6 \%. Hier ist jedoch der Wegfall der 2007 im Rahmen der Tarifeinigung zu T-Service vereinbarten Ausgleichszahlungen von jeweils 2,2\% gegenzurechnen (Bispinck/WSI-Tarifarchiv 2008), sodass effektiv ein Plus von insgesamt 4,2 \% verbleibt. Die Laufzeit endet jeweils zum 31.12.2010.

Den ersten großen Flächenabschluss gab es am 1.3.2009 im öffentlichen Dienst für die Länder (ohne Hessen und Berlin). Er sieht die Anhebung der Tarifentgelte um einheitlich $40 €$ sowie um $3 \%$ ab 1.3.2009 vor. Am 1.3.2010 folgt eine Stufenerhöhung um 1,2 \% mit einer Laufzeit bis 31.12. 2010. Damit konnte ver.di im Wesentlichen die Einkommensentwicklung bei Bund und Gemeinden nachvollziehen. 


\begin{tabular}{|c|c|c|}
\hline \multicolumn{3}{|c|}{$\begin{array}{l}\text { Übersicht 2: Ausgewählte Lohn- und Gehaltsabschlüsse West und Ost } \\
\text { für } 2009\end{array}$} \\
\hline Abschluss & Tarifbereich & Ergebnis \\
\hline 12.11 .2008 & $\begin{array}{l}\text { Metallindustrie } \\
\text { Baden-Württemberg } \\
\text { (Pilotabschluss) }\end{array}$ & $\begin{array}{l}510 € \text { Pauschale insg. für November 2008-Januar } 2009 \\
\mathbf{2 , 1} \% \text { ab 01.02.09 } \\
\mathbf{2 , 1} \% \text { ab 01.05.09 (auf Basis des Entgelts Nov. 2008) } \\
122 € \text { Einmalzahlung im September 2009, Laufzeit bis } \\
30.04 .10 \\
0,4 \% \text { Einmalzahlung jeweils für Januar-April } 2010 \\
\text { (Finanzierung Altersteilzeit) }\end{array}$ \\
\hline 12.12 .2008 & Tarifgruppe RWE & $\begin{array}{l}\mathbf{4 , 0} \% \text { ab } 01.01 .09 \\
\mathbf{2 , 2} \% \text { Stufenerhöhung ab } 01.01 .10 \\
1.200 € \text { Einmalzahlung für } 2009 \text { und weitere } 800 € \text { für } 2010 \\
\text { Laufzeit bis } 31.10 .10\end{array}$ \\
\hline 31.01 .2009 & Deutsche Bahn AG & $\begin{array}{l}\mathbf{2 , 5} \% \text { ab 01.02.09 } \\
\mathbf{2 , 0} \% \text { Stufenerhöhung ab 01.01.10 } \\
500 € \text { Einmalzahlung im Dezember 2009, Laufzeit bis } \\
\text { 31.07.10 }\end{array}$ \\
\hline 01.03 .2009 & $\begin{array}{l}\text { Öffentlicher Dienst } \\
\text { Länder }\end{array}$ & $\begin{array}{l}40 € \text { Pauschale insg. für Januar und Februar } 2009 \\
40 € \text { Sockelbetrag ab 01.03.09 } \\
3,0 \% \text { ab 01.03.2009 } \\
1,2 \% \text { Stufenerhöhung ab 01.03.10, Laufzeit bis } 31.12 .10\end{array}$ \\
\hline 10.03.2009 & $\begin{array}{l}\text { Textil- und Bekleidungs- } \\
\text { industrie West }\end{array}$ & $\begin{array}{l}\text { nach } 2 \text { Nullmonaten (März und April) } \\
340 € \text { Pauschale insg. für Mai-Dezember } \\
1,5 \% \text { sowie } 40 € \text { Sockelbetrag ab } 01.01 .10 \\
99 € \text { Einmalzahlung insg. für Januar und Februar 2011, } \\
\text { Laufzeit bis 28.02.11 }\end{array}$ \\
\hline 13.03.2009 & Deutsche Telekom AG & $\begin{array}{l}\mathbf{3}, 0 \% \text { ab 01.01.09 } \\
\mathbf{2 , 5} \% \text { Stufenerhöhung ab 01.01.10, Laufzeit bis 31.12.10 }\end{array}$ \\
\hline 01.04 .2009 & $\begin{array}{l}\text { Eisen- und Stahlindustrie } \\
\text { Niedersachsen, Bremen, } \\
\text { Nordrhein-Westfalen }\end{array}$ & $\begin{array}{l}350 € \text { Pauschale insg. für April-Dezember } 2009 \\
\mathbf{2 , 0} \% \text { ab 01.01.10, Laufzeit bis } 31.08 .10\end{array}$ \\
\hline 22.04 .2009 & Bankgewerbe & $\begin{array}{l}\text { nach } 4 \text { Nullmonaten (Juli-Oktober } 2008 \text { ) } \\
2,5 \% \text { ab 01.11.08 } \\
200 € \text { Einmalzahlung im Februar 2010, Laufzeit bis } 30.04 .10\end{array}$ \\
\hline 12.05 .2009 & $\begin{array}{l}\text { Süßwarenindustrie } \\
\text { Nordrhein-Westfalen }\end{array}$ & $\begin{array}{l}\text { nach } 2 \text { Nullmonaten (April und Mai) } \\
3,0 \% \text { ab 01.06.09 } \\
1,9 \% \text { Stufenerhöhung ab 01.04.10, Laufzeit bis } 31.03 .11\end{array}$ \\
\hline 18.05 .2009 & $\begin{array}{l}\text { Energie- und Versorgungs- } \\
\text { wirtschaft Ost (AVEU) }\end{array}$ & $\begin{array}{l}100 € \text { Pauschale für Mai } \\
3,62 \% \text { ab 01.06.09, Laufzeit bis } 30.06 .10\end{array}$ \\
\hline 23.05 .2009 & Bauhauptgewerbe & $\begin{array}{l}60 € \text { Pauschale insg. für April und Mai } \\
\text { West und Berlin: } \\
\text { 2,3\% ab 01.06.09 } \\
\text { 2,3\% Stufenerhöhung ab 01.04.10, Laufzeit bis 31.03.11 } \\
\text { Ost: Anhebung um Cent-Werte der West-Anhebung } \\
\text { stufenweise Anhebung der Mindestlöhne }\end{array}$ \\
\hline 03.06.2009 & Druckindustrie & $\begin{array}{l}280 € \text { Pauschale insg. für April 2009-März } 2010 \\
\mathbf{2 , 0} \% \text { ab 01.04.10, Laufzeit bis 31.03.11 }\end{array}$ \\
\hline 04.06 .2009 & Versicherungsgewerbe & $\begin{array}{l}250 € \text { Pauschale insg. für Oktober 2009-März } 2010 \\
2,5 \% \text { ab 01.04.10, Laufzeit bis 31.03.11 }\end{array}$ \\
\hline 11.06 .2009 & $\begin{array}{l}\text { Einzelhandel } \\
\text { Nordrhein-Westfalen }\end{array}$ & $\begin{array}{l}\text { nach } 4 \text { Nullmonaten (Mai-August) } \\
2,0 \% \text { ab 01.09.09 } \\
1,5 \% \text { Stufenerhöhung ab 01.09.10 } \\
150 € \text { zusätzliche Einmalzahlung im April 2010, Laufzeit bis } \\
30.04 .11\end{array}$ \\
\hline 27.07.2009 & $\begin{array}{l}\text { Sozial- und } \\
\text { Erziehungsdienst }\end{array}$ & $\begin{array}{l}\text { neue einheitliche Entgelttabelle mit } 16 \text { Entgeltgruppen } \\
\text { Entgeltbeträge zwischen } 1.750-4.525 €\end{array}$ \\
\hline 29.10 .2009 & $\begin{array}{l}\text { Gebäudereiniger- } \\
\text { handwerk (Arb.) }\end{array}$ & $\begin{array}{l}\text { nach } 3 \text { Nullmonaten (Oktober-Dezember) } \\
3,1 / 3,8-6,2 \% \text { regional unterschiedlich (West inkl. } \\
\text { Berlin/Ost) ab 01.01.10 } \\
\text { 1,8/2,5\% (West inkl. Berlin/Ost) Stufenerhöhung ab } \\
01.01 .11, \text { Laufzeit bis 31.12.11 }\end{array}$ \\
\hline
\end{tabular}

Quelle: WSI-Tarifarchiv, Stand: Dezember 2009.

WSI MITTELUUNGEN

In der Textil- und Bekleidungsindustrie (West) sah der Abschluss vom 10.3.2009 nach zwei Nullmonaten eine Pauschalzahlung für Mai bis Dezember in Höhe von insgesamt $340 €$ vor. Ab 1.1.2010 folgt eine Tarifanhebung um 1,5 \%, ein Sockelbetrag von $40 €$ sowie eine zusätzliche Einmalzahlung von $99 €$ für Januar und Februar 2011 bei einer Laufzeit bis 28.2.2011. Auch
2009 bis März 2010 und einer Tarifsteigerung von $2 \%$ ab 1.4.2010 bei einer Laufzeit bis 31.3.2011.

Anders in der Süßwarenindustrie, wo die Gewerkschaft Nahrung, Genuss, Gaststätten (NGG) in Nordrhein-Westfalen einen bemerkenswerten Abschluss erzielen konnte. Er sieht nach zwei Nullmonaten eine Tarifanhebung um $3 \%$ ab dem 1.6.2009 sowie eine Stufenerhöhung von 1,9\% ab dem 1.4.2010 bei einer Laufzeit bis zum 31.3.2011 vor. Gleichlautende Abschlüsse gab es auch in den anderen regionalen Tarifbereichen.

Im Bauhauptgewerbe (West) vereinbarten die Tarifparteien am 23.5.2009 einen Abschluss mit einer Pauschalzahlung von $60 €$ für April und Mai 2009, einer anschließenden Tariferhöhung von 2,3\% und einer Stufenanhebung von erneut 2,3\% ab 1.4.2010 bei einer Laufzeit bis 31.3.2011. Im Osten fällt die Anhebung etwas höher aus. Die Mindestlöhne werden stufenweise angehoben.

Im Einzelhandel erreichte ver.di am 11.6.2009 in Nordrhein-Westfalen einen ersten Abschluss, der auch zur Übernahme in den anderen Tarifbereichen empfohlen wurde: Nach vier Nullmonaten gibt es ab dem 1.9.2009 eine Tarifanhebung um $2 \%$ und zum 1.9.2010 eine Stufenanhebung um weitere $1,5 \%$ bei einer Laufzeit bis zum 30.4.2011. Im April 2010 erfolgt eine Einmalzahlung von $150 €$.

Im Versicherungsgewerbe sah die Tarifvereinbarung vom 4.6.2009 eine Pauschalzahlung von $250 €$ für Oktober 2009 bis März 2010 sowie eine Tarifanhebung von 2,5 \% mit einer Laufzeit bis Ende März 2011 vor.

Im Groß- und Außenhandel vereinbarten die regionalen Tarifparteien im Oktober und November überwiegend nach fünf Nullmonaten eine Tarifsteigerung von zunächst 2,0\%, nach acht Monaten eine Stufenanhebung von weiteren $1,5 \%$ sowie eine zusätzliche Einmalzahlung für die unteren Vergütungsgruppen bei einer Gesamtlaufzeit von zwei Jahren.

Nach Warnstreiks und regulärem Arbeitskampf setzte die IG BAU im Gebäudereinigerhandwerk nach drei Nullmonaten eine Tarifanhebung von $3,1 / 3,8 \%$ bis $6,2 \%$ regional unterschiedlich (West inkl. Berlin/Ost) ab 1.1.2010 und eine weitere Stufenanhebung nach zwölf Monaten von 1,8/2,5 \% durch. Außerdem wurde ein Einstieg zur Regelung einer tariflichen Zusatzrente vereinbart. 
2

\section{Tarifentwicklung}

\subsection{LOHN- UND GEHALT}

Die DGB-Gewerkschaften schlossen 2009 in ganz Deutschland Lohn- und Gehaltstarifverträge für 7,9 Mio. Beschäftigte ab, davon für rund 6,8 Mio. in den alten und 1,1 Mio. in den neuen Bundesländern. Das entspricht rund $41 \%$ der von Tarifverträgen erfassten Beschäftigten. Für weitere 7,9 Mio. Beschäftigte traten Stufenerhöhungen in Kraft, die bereits 2008 oder früher vereinbart worden waren. Bei rund 3,3 Mio. Beschäftigten liefen 2008 oder früher die Vergütungstarifverträge aus, aber es kam bis zum Jahresende (noch) nicht zu Neuabschlüssen bzw. es traten keine Tarifanhebungen in Kraft.

\section{ABSCHLUSSRATE}

Die tarifliche Abschlussrate belief sich gesamtwirtschaftlich im Durchschnitt auf 3,9\% (2008: 5,2\%), in Westdeutschland betrug die Rate 3,8\%, in Ostdeutschland 4,4\%. Die Abschlussrate schließt alle, ggf. auch 2010 und später in Kraft tretende tabellenwirksamen Erhöhungen ein. Nicht berücksichtigt werden Pauschalzahlungen und zusätzliche Einmalzahlungen, die sich nicht dauerhaft in den Tariftabellen niederschlagen. Die Spannweite der durchschnittlichen Gesamtabschlussraten reicht von 2,5\% im Bereich Kreditinstitute/Versicherungsgewerbe, 2,9\% im Verbrauchsgütergewerbe, $3,3 \%$ im Grundstoff- und Produktionsgütergewerbe, 4,7 \% im Bereich private Dienstleistungen/Organisationen ohne Erwerbszweck, 5,5 \% im Bereich Gebietskörperschaften, Sozialversicherung bis zu 5,9 \% im Bereich Energieund Wasserversorgung, Bergbau.

Diese Gesamtabschlussraten sind nur von begrenzter Aussagekraft, weil sie sich immer auf die gesamte, je nach Tarifbereich sehr unterschiedlich lange Laufzeit der Tarifabkommen beziehen. Berücksichtigt man lediglich die im Jahr 2009 abgeschlossenen und auch in Kraft getretenen Tariferhöhungen, ergibt sich eine Abschlussrate von 2,4 \% (West: 2,4 \%, Ost: 2,8 \%). Differenziert man diese Größe nach Wirtschaftsbereichen, dann ergibt sich für 2009 eine Streuung zwischen 1,7 \% im Verbrauchs-

Tabelle 1: Laufzeit der Tarifverträge - in Monaten -

\begin{tabular}{lcccccccccc}
\hline & $\mathbf{2 0 0 0}$ & $\mathbf{2 0 0 1}$ & $\mathbf{2 0 0 2}$ & $\mathbf{2 0 0 3}$ & $\mathbf{2 0 0 4}$ & $\mathbf{2 0 0 5}$ & $\mathbf{2 0 0 6}$ & $\mathbf{2 0 0 7}$ & $\mathbf{2 0 0 8}$ & $\mathbf{2 0 0 9}$ \\
\hline Gesamt & 21,8 & 14,5 & 18,3 & 20,5 & 21,8 & 25,7 & 22,1 & 22,2 & 22,4 & 24,1 \\
\hline West & 21,5 & 14,1 & 18,1 & 20,4 & 21,8 & 25,2 & 21,6 & 22,2 & 22,2 & 24,3 \\
\hline Ost & 23,3 & 16,4 & 19,7 & 21,0 & 22,0 & 28,4 & 24,7 & 21,9 & 23,4 & 23,2 \\
\hline \multicolumn{1}{l}{ Quelle: WSI-Tarifarchiv, Stand: 31.12 .2009}$.
\end{tabular}

gütergewerbe und 4,2\% im Bereich Gebietskörperschaften/Sozialversicherung. $\mathrm{Zu}$ berücksichtigen ist stets, dass die Erhöhungen zu unterschiedlichen Zeitpunkten im Jahresverlauf wirksam wurden.

Wie bereits in den Vorjahren spielten auch im Jahr 2009 „Nullmonate“ bei den Tarifabschlüssen eine bedeutende Rolle. Für rund 7,3 Mio. (2008: 7,9 Mio.), das entspricht rund $92 \%$ der von Neuabschlüssen begünstigten Beschäftigten, gab es Tarifabschlüsse mit verzögerter Anpassung der Lohn- und Gehaltserhöhungen. 28 \% mussten zwischen ein bis drei Monate auf die reguläre Tariferhöhung warten, für weitere $44 \%$ vergingen vier bis fünf Monate bis zur ersten Tarifsteigerung, $20 \%$ mussten sogar sechs und mehr Nullmonate akzeptieren. Nur für $36 \%$ der davon betroffenen Beschäftigten vereinbarten die Gewerkschaften als Ausgleich Pauschalzahlungen, die durchschnittlich $35 €$ (West: $37 €$, Ost: $30 €)$ im Monat betrugen.

\section{LAUFZEITEN}

Der seit rund zehn Jahren zu beobachtende Trend zu längeren Laufzeiten hat sich im vergangenen Jahr fortgesetzt. Die Laufzeit der Vergütungstarifverträge im Jahr 2009 beträgt durchschnittlich 24,1 Monate (2008: 22,4 Monate). Für rund 0,6 Mio. Beschäftigte $(7,2 \%)$ laufen die Abkommen zwischen zwölf und 17 Monaten, für knapp
1 Mio. (12,5\%) 18 bis 23 Monate, für 6,3 Mio. (80,2 \%) 24 Monate und länger. In den neuen Bundesländern laufen die Tarifverträge im Schnitt rund einen Monat kürzer als in den alten.

\section{JAHRESBEZOGENE TARIFSTEIGERUNG}

Bei der jahresbezogenen Steigerung der tariflichen Grundlöhne und -gehälter werden im Unterschied zur tariflichen Abschlussrate die Auswirkungen aus der (oft unterschiedlichen) Lage und Laufzeit der Tarifabkommen berücksichtigt. Auch werden gegebenenfalls im Berichtsjahr wirksam werdende Abschlüsse aus den Vorjahren sowie zusätzliche Einmalzahlungen und Pauschalzahlungen als Ausgleich für Abschlussverzögerungen mit einbezogen. Die jahresbezogene Tarifsteigerung setzt die durchschnittliche tarifliche Grundvergütung des gesamten Jahres 2009 zum Vorjahr in Bezug und erfasst insgesamt 15,8 Mio. Arbeitnehmerinnen und Arbeitnehmer.

Diese kalenderjährliche Steigerung der Tarifverdienste 2009 gegenüber 2008 betrug für ganz Deutschland 2,6 \% (2008: 2,9\%) (Tabelle 2). Am höchsten fiel die jahresbezogene Tarifsteigerung mit 4,1\% im Bereich Energie- und Wasserversorgung, Bergbau aus, gefolgt vom Bereich Gebietskörperschaften, Sozialversicherung mit 3,7 \% und dem Investitionsgütergewerbe mit 3,3\%. Unterdurchschnittlich war die
Tabelle 2: Tarifsteigerung $2009^{1}$ - in \% -

\begin{tabular}{lccc} 
Wirtschaftsbereich & Ost & West & Gesamt \\
\hline Gartenbau, Land- und Forstwirtschaft & 3,6 & 2,4 & 2,8 \\
Energie- und Wasserversorgung, Bergbau & 3,6 & 4,2 & 4,1 \\
Grundstoff- und Produktionsgütergewerbe & 3,5 & 2,4 & 2,5 \\
Investitionsgütergewerbe & 3,4 & 3,3 & 3,3 \\
Verbrauchsgütergewerbe & 2,8 & 2,3 & 2,4 \\
Nahrungs- und Genussmittelgewerbe & 2,8 & 2,3 & 2,4 \\
Baugewerbe & 2,5 & 2,3 & 2,4 \\
Handel & 1,4 & 1,4 & 1,4 \\
Verkehr und Nachrichtenübermittlung & 3,8 & 2,8 & 2,9 \\
Kreditinstitute, Versicherungsgewerbe & 1,8 & 1,8 & 1,8 \\
Priv. Dienstleistungen, Organ. o. Erwerbszweck & 1,9 & 2,0 & 1,9 \\
Gebietskörperschaften, Sozialversicherung & 4,4 & 3,6 & 3,7 \\
\hline Gesamte Wirtschaft & $\mathbf{3 , 0}$ & $\mathbf{2 , 6}$ & $\mathbf{2 , 6}$ \\
\hline 1) Jahresbezogene Erhöhung der tariflichen Grundvergütung 2009 gegenüber 2008. & WSI MITTEILUNGEN \\
Quelle: WSI-Tarifarchiv, Stand: 31.12.2009. & & &
\end{tabular}


Tabelle 3: Tarifliche Wochen- und Jahresarbeitszeit 2000-2009 - in Std. -

\begin{tabular}{|c|c|c|c|c|c|c|c|c|c|c|c|}
\hline & & 2000 & 2001 & 2002 & 2003 & 2004 & 2005 & 2006 & 2007 & 2008 & 2009 \\
\hline \multirow[t]{3}{*}{ Woche } & $G$ & 37,7 & 37,7 & 37,7 & 37,7 & 37,6 & 37,6 & 37,6 & 37,6 & 37,6 & 37,7 \\
\hline & W & 37,4 & 37,4 & 37,4 & 37,4 & 37,4 & 37,4 & 37,4 & 37,4 & 37,4 & 37,4 \\
\hline & $\mathrm{O}$ & 39,1 & 39,1 & 39,1 & 39,0 & 38,9 & 39,0 & 38,9 & 38,8 & 38,8 & 38,8 \\
\hline \multirow[t]{3}{*}{ Jahr } & $G$ & 1657,8 & 1656,3 & 1656,0 & 1656,3 & 1655,7 & 1655,6 & 1657,0 & 1656,8 & 1656,2 & 1657,8 \\
\hline & W & 1642,5 & 1641,9 & 1642,6 & 1643,5 & 1643,3 & 1643,2 & 1644,7 & 1645,2 & 1644,8 & 1646,7 \\
\hline & $\mathrm{O}$ & 1727,7 & 1724,2 & 1722,7 & 1721,9 & 1719,2 & 1718,9 & 1720,3 & 1716,4 & 1715,4 & 1715,7 \\
\hline \multicolumn{8}{|c|}{ Stand: jeweils in Kraft zum 31.12. jeden Jahres. $G=$ Gesamt, $W=$ West, $O=$ Ost } & & & & \\
\hline
\end{tabular}

\begin{tabular}{|c|c|c|c|}
\hline \multicolumn{4}{|c|}{ Übersicht 3: Tarifliche Arbeitszeitregelungen 2009} \\
\hline Tarifregelung & Ost & West & Gesamt \\
\hline Wochenarbeitszeit (Std.) & 38,8 & 37,4 & 37,7 \\
\hline \multicolumn{4}{|l|}{ Anteil der Beschäftigten (in \%) mit: } \\
\hline bis zu 35 & 5,9 & 25,1 & 21,9 \\
\hline $36-37$ & 5,4 & 9,4 & 8,7 \\
\hline $37,5-38,5$ & 31,2 & 36,7 & 35,6 \\
\hline $39-40$ und mehr & 57,0 & 28,4 & 33,0 \\
\hline Urlaub (Arbeitstage) ${ }^{1)}$ & 29,5 & 30,1 & 30,0 \\
\hline Jahresarbeitszeit (Std.) & $1.715,7$ & $1.646,7$ & $1.657,8$ \\
\hline $\begin{array}{l}\text { 1) Endstufe. } \\
\text { Quelle: WSI-Tarifarchiv, Stand: } 31.12 .2009 .\end{array}$ & & $M$ & ILUNGEI \\
\hline
\end{tabular}

Tarifsteigerung in den Bereichen Verbrauchsgütergewerbe, Nahrungs- und Genussmittelgewerbe und Baugewerbe mit jeweils 2,4 \%, im Bereich private Dienstleistungen, Organisationen ohne Erwerbszweck mit $1,9 \%$ und in den Bereichen Kreditinstitute, Versicherungsgewerbe mit $1,8 \%$. Am niedrigsten fiel die Steigerung im Handel mit 1,4 \% aus. In Ostdeutschland lag die kalenderjährliche Erhöhung mit 3,0\% höher als in Westdeutschland mit 2,6\%.

Positiv beeinflusst wird die jahresbezogene Tarifsteigerung 2009 durch die länger laufenden Abschlüsse aus 2008. Die daraus resultierende Tarifanhebung für 2009 beläuft sich auf 3,3\%, die Neuabschlüsse des Jahres 2009 ergeben lediglich 2,0 \%. Im Mittel ergeben sich dann die bereits genannten $2,6 \%{ }^{1}$

In Tarifbereichen mit knapp 1 Mio. Beschäftigten liefen Vergütungstarifverträge im Jahr 2009 aus, ohne dass bis zum Jahresende neue Abschlüsse getätigt wurden. Bezieht man diese Tarifbereiche mit ein, dann sinkt die jahresbezogene Tarifsteigerung geringfügig von $2,6 \%$ auf $2,5 \%$. In Tarifbereichen mit weiteren 2,3 Mio. Beschäftigten sind die Tarifverträge bereits 2008 oder in den Jahren zuvor ausgelaufen ohne nachfolgende Abschlüsse. Berücksichtigt man auch diese Bereiche, dann sinkt die jahresbezogene Tarifsteigerung für 2009 insgesamt auf 2,3\%.

Die durchschnittliche jahresbezogene Tarifsteigerung 2009 von 2,6 \% liegt deut-

(West: $3,3 \%$, Ost: $4,9 \%$ ), der weit über der Steigerung der tariflichen Grundvergütungen und auch oberhalb des Anstiegs der Verbraucherpreise liegt (BIBB 2009). Je nach Tarifbereich verbergen sich hinter diesen Durchschnittszahlen große Unterschiede: Gemessen an der Ausbildungsvergütung im dritten Ausbildungsjahr lagen die Beträ- ge Ende 2009 in acht der für diesen Tarifbericht ausgewählten 26 Tarifbereiche unverändert auf dem Vorjahresniveau. Im Übrigen variierten die Anhebungen zwischen $0,6 \%$ im Versicherungsgewerbe und 8,2\% im öffentlichen Dienst (Länder).

\subsection{ARBEITSZEIT}

Die gesamtwirtschaftlichen Eckdaten der tariflichen Wochenarbeitszeit sind im Laufe des vergangenen Jahres weitgehend gleich geblieben (Tabelle 3). Die tarifliche Wochenarbeitszeit betrug Ende 2009 im gesamtwirtschaftlichen Durchschnitt in ganz Deutschland 37,7 Stunden (West: 37,4; Ost: 38,8 Stunden) (Übersicht 3). Angesichts der zahlreichen arbeitszeitbezogenen Öffnungsklauseln und Flexi-Bestimmungen (u.a. mit Kontenregelungen und langen Ausgleichszeiträumen) müssen die gesamtwirtschaftlichen und branchenbezogenen Zahlen zur tariflichen Arbeitszeitdauer eher als Referenzgrößen denn als Beschreibung der tatsächlichen Arbeitszeitstandards angesehen werden.

Die tarifliche Urlaubsdauer (Endstufe) beträgt im gesamtdeutschen Durchschnitt unverändert 30,0 Tage (West: 30,1; Ost: 29,5 Tage).

Errechnet man auf Basis dieser und weiterer Einzelkomponenten die tarifliche Jahresarbeitszeit, so ergibt sich ein gesamtdeutscher Durchschnitt von 1.657,8 Stunden, für Westdeutschland 1.646,7 und für Ostdeutschland 1.715,7 Stunden.

\subsection{TARIFBINDUNG UND TARIFLOSE ZUSTÄNDE}

Seit Jahren ist die Tarifbindung in ganz Deutschland leicht aber beständig rückläufig (Ellguth/Kohaut 2008). Nach den Ergebnissen des IAB-Panels arbeiteten im Jahr 2008 rund $63 \%$ der Beschäftigten in Westdeutschland und $52 \%$ derjenigen in Ostdeutschland in tarifgebundenen Betrieben (2007: 63/54 \% in West/Ost). Im gesamtdeutschen Durchschnitt ergibt das $61 \%$. Im Jahr 1998 betrug die gesamtdeutsche Tarifbindung noch $74 \%$. Tabelle 4 zeigt die erheblichen branchenbezogenen
Wertet man den Metallabschluss vom November 2008 als quasi ersten Abschluss der Tarifrunde sich die genannten Tarifsteigerungen auf 3,0 \% (2008er Abschlüsse) und 2,5\% (2009er Abschlüsse). 2009 und schlägt inn diesem Jahr zu, verändern 
Unterschiede: von $43 \%$ in der Landwirtschaft über $65 \%$ im Bereich des Investitionsgütergewerbes bis zu $98 \%$ im Bereich der öffentlichen Verwaltung.

Die Ursachen des Rückgangs liegen zum einen darin, dass vormals tarifgebundene Unternehmen die Tarifbindung insgesamt aufgeben oder durch Aufspaltung und Outsourcing Teile des Unternehmens aus der Tarifbindung herauslösen. Zum anderen vermeiden neu gegründete Unternehmen häufig einen Beitritt zum Arbeitgeberverband und schließen auch keine Firmentarifverträge ab.

Ein dritter Grund für die schwächere Tarifbindung liegt darin, dass die Gewerkschaften in einer ganzen Reihe von Tarifbereichen nicht in der Lage sind, nach Auslaufen der Tarifverträge neue Tarifabkommen abzuschließen. Je länger dieser tariflose Zustand anhält, umso mehr verlieren die alten Tarifregelungen an praktischer Bedeutung. Eine aktuelle Analyse des WSITarifarchivs ergibt, dass nach wie vor über 50 Tarifbranchen mit rund 2,3 Mio. Beschäftigten aus dem Handwerk, dem Dienstleistungssektor, aber auch aus der Industrie mindestens seit dem Jahr 2008, größtenteils aber seit zwei, drei und mehr Jahren ohne Neuabschluss sind.

In einigen wenigen Bereichen ist aber auch eine Wiederherstellung der Tarifbindung gelungen, zumeist erst nach mehreren Jahren, so beispielsweise in der Floristik, in einigen Bereichen des Steinmetzund Steinbildhauerhandwerks sowie des Bäcker- und Konditorenhandwerks.

\section{3 \\ Effektivverdienst- entwicklung}

\subsection{GESAMTWIRTSCHAFTLICHE ENTWICKLUNG}

Die Effektiveinkommens- und Verteilungsentwicklung in Deutschland hat krisenbedingt einen außergewöhnlichen Verlauf genommen (vgl. Statistisches Bundesamt 2010): Die Summe der Bruttolöhne und -gehälter ist 2009 um 0,5\% zurückgegangen. Je beschäftigten Arbeitnehmer ergibt sich auf Monatsbasis bereits nominal ein Rückgang um 0,4 \%. Daraus folgt zugleich, dass die Bruttoreallöhne 2009 (zum sechsten Mal in Folge) real - und zwar um 0,8 \% - gesunken sind.
Tabelle 4: Tarifbindung der Beschäftigten in Deutschland 2008 - Anteile der Beschäftigten in \% -

\begin{tabular}{lccc} 
& West & Ost & Gesamt \\
\hline Öffentliche Verwaltung & 98 & 96 & 98 \\
Bergbau/Energie & 93 & 88 & 92 \\
Kredit/Versicherung & 87 & 71 & 85 \\
Baugewerbe & 74 & 57 & 70 \\
Grundstoffverarbeitung (Produktionsgüter) & 72 & 43 & 68 \\
Investitionsgüter & 68 & 41 & 65 \\
\hline Gesamt & 63 & 52 & 61 \\
\hline Verbrauchsgüter & 64 & 42 & 61 \\
sonstige Dienste & 63 & 53 & 61 \\
Org. ohne Erwerbszweck & 62 & 42 & 58 \\
Verkehr/Nachrichten & 56 & 48 & 55 \\
Handel/Reparatur & 51 & 30 & 47 \\
Dienste für Unternehmen & 43 & 51 & 45 \\
Landwirtschaft u.a. & 54 & 17 & 43 \\
\hline & & W SI MITTEILUNGEN
\end{tabular}

Auf Stundenbasis berechnet, sieht das Bild anders aus. Da die Betriebe durch Kurzarbeit und andere Maßnahmen die Beschäftigung relativ stabilisiert haben und den Personalstand nicht entsprechend dem Produktionsrückgang heruntergefahren haben, sind die Verdienste je Arbeitnehmerstunde um 2,9\% gestiegen (Tabelle 5). Die Kehrseite der Medaille ist die sinkende Arbeitsproduktivität. Sie ist 2009 je Erwerbstätigen um 4,9\% und je Erwerbstätigenstunde um 2,2\% zurückgegangen. Dies hat Konsequenzen für die Lohnstückkosten, also das Verhältnis von Lohnkosten zur Arbeitsproduktivität. Pro Kopf sind die Lohnstückkosten um 5,1\%, pro Stunde um 5,6 \% gestiegen (Tabelle 5). Stärker sind die Lohnstückkosten zuletzt im Jahr 1992 $(+6,3 \%)$ angestiegen. Dieser starke Anstieg ist im Wesentlichen eine Folge des massiven Rückgangs des Bruttoinlandsprodukts vor allem im IV. Quartal 2008 und im I. Quartal 2009. Im II. Quartal hat sich die Entwicklung schon wieder abgeflacht. „Sorgen über Deutschlands preisliche Wettbewerbsfähigkeit sind aber völlig unnötig, wie der Vergleich der Lohnstückkostenzuwächse mit anderen großen Län- dern des Euroraums ... zeigt." (IMK 2009, S. 11).

\subsection{ABSTRICHE BEI BETRIEBLICHEN ENTGELTEN}

Die große Lücke zwischen Tarif- und Effektivlohnentwicklung im Jahr 2009 ist maßgeblich auf die betriebliche Krisenverarbeitung zurückzuführen. In einer repräsentativen Betriebsrätebefragung hat das WSI die unterschiedlichen Reaktionen und betrieblichen Maßnahmen zur Krisenbewältigung analysiert (Bogedan et al. 2009). Befragt wurden 2.324 Betriebsräte aus Betrieben mit 20 und mehr Beschäftigten im Zeitraum von Mitte Juli bis Ende September 2009. Im Ergebnis zeigt sich, dass bei der Krisenbewältigung und Beschäftigungssicherung vor allem arbeitszeitbezogene Maßnahmen eine zentrale Rolle spielten. An erster Stelle standen dabei der Abbau von Arbeitszeitkonten (bzw. der Aufbau von Zeitschulden) und die Kurzarbeit, gefolgt von betrieblichen Umund Versetzungen und Maßnahmen zur Urlaubsnahme. Erst an fünfter Stelle folgten Abstriche beim Entgelt und als Letztes

\section{Tabelle 5: Wirtschafts- und Verteilungsdaten* 2007-2009}

- Veränderung gegenüber dem Vorjahr in \% -

\begin{tabular}{lccr}
\hline & $\mathbf{2 0 0 7}$ & $\mathbf{2 0 0 8}$ & $\mathbf{2 0 0 9}$ \\
\hline Bruttoinlandsprodukt & 2,5 & 1,3 & $-5,0$ \\
Verbraucherpreise & 2,2 & 2,6 & 0,4 \\
Produktivität/Stunde & 0,7 & 0,0 & $-2,2$ \\
Tarifentgelte/Stunde* & 2,2 & 2,9 & 2,6 \\
Bruttoverdienste/Stunde & 1,4 & 2,3 & 2,9 \\
Lohnstückkosten/Stunde & 0,1 & 2,2 & 5,6 \\
\hline * je tarifliche Arbeitstunde & & WSI MITTEILUNGEN \\
Quelle: Stat. Bundesamt, WSI-Tarifarchiv. & & &
\end{tabular}


Tabelle 6: Betriebliche Maßnahmen in der Krise - Angaben der Betriebsräte in \%, Mehrfachnennungen -

\begin{tabular}{|c|c|c|c|c|c|c|c|c|}
\hline & 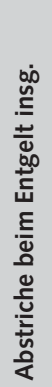 & 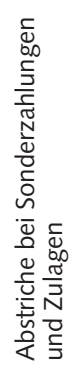 & 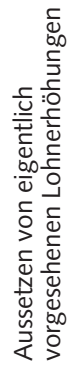 & 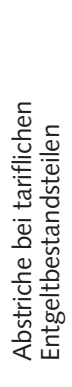 & 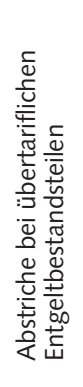 & 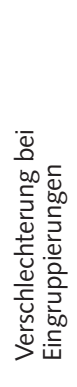 & 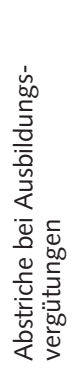 & 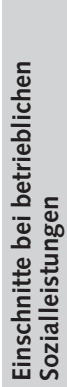 \\
\hline Alle Betriebe & 16 & 11 & 9 & 8 & 6 & 5 & 1 & 8 \\
\hline \multicolumn{9}{|l|}{$\begin{array}{l}\text { Betriebe seit Mitte } 2008 \text { von Krise } \\
\text { betroffen }\end{array}$} \\
\hline ja & 22 & 15 & 13 & 10 & 9 & 6 & 1 & 10 \\
\hline nein & 10 & 6 & 4 & 5 & 3 & 4 & 1 & 5 \\
\hline Betriebe mit starker Exportabhängigkeit & 20 & 13 & 13 & 9 & 7 & 5 & 1 & 7 \\
\hline West & 17 & 11 & 10 & 8 & 7 & 5 & 1 & 8 \\
\hline Ost & 13 & 9 & 6 & 6 & 3 & 5 & 1 & 6 \\
\hline \multicolumn{9}{|l|}{ Betriebsgröße } \\
\hline bis 100 Beschäftigte & 15 & 10 & 9 & 8 & 5 & 5 & 1 & 6 \\
\hline 101 bis 500 & 17 & 12 & 9 & 7 & 7 & 5 & 2 & 9 \\
\hline 501 bis 1000 & 15 & 11 & 7 & 5 & 6 & 6 & 1 & 15 \\
\hline über 1000 & 19 & 11 & 11 & 11 & 11 & 2 & 0 & 8 \\
\hline Grundstoffe/Produktionsgüter & 22 & 15 & 10 & 9 & 9 & 5 & 1 & 6 \\
\hline Investitions- und Gebrauchsgüter & 21 & 13 & 14 & 8 & 6 & 4 & 1 & 6 \\
\hline Verbrauchsgüter & 20 & 13 & 11 & 14 & 8 & 8 & 3 & 9 \\
\hline Baugewerbe & 15 & 8 & 9 & 11 & 6 & 5 & 0 & 4 \\
\hline Handel (und Reparatur) & 15 & 12 & 8 & 6 & 7 & 4 & 2 & 10 \\
\hline Verkehr und Nachrichten & 13 & 5 & 6 & 5 & 8 & 6 & 1 & 7 \\
\hline Kredit und Versicherungen & 13 & 13 & 6 & 1 & 11 & 6 & 1 & 10 \\
\hline $\begin{array}{l}\text { sonstige priv. und öffentl. } \\
\text { Dienstleistungen }\end{array}$ & 14 & 9 & 8 & 8 & 4 & 5 & 1 & 8 \\
\hline sonstige Branchen & 2 & 1 & 0 & 0 & 0 & 1 & 0 & 2 \\
\hline
\end{tabular}

Einschnitte bei betrieblichen Sozialleistungen.

Im Folgenden sollen vor allem die unmittelbar entgeltbezogenen Maßnahmen der Betriebe näher beleuchtet werden. Rund $16 \%$ der Betriebsräte geben an, dass in ihrem Betrieb Abstriche beim Entgelt erfolgt sind oder geplant waren (Tabelle 6). ${ }^{2}$ Allerdings zeigen sich z. T. deutliche Unterschiede: So steigt der Anteil der Betriebe mit Abstrichen beim Entgelt bei Großbetrieben auf $19 \%$ (ab 1.000 Beschäftigte). Betroffen sind vor allem Branchen aus den Bereichen Grundstoffe/Produktionsgüter (22\%), Verbrauchsgüter $(21 \%)$ und Investitions- und Gebrauchsgüter (20\%). Weniger betroffen sind Betriebe aus den Bereichen Verkehr und Nachrichten sowie Kredit und Versicherungen (jeweils $13 \%$ ). Betriebe im Westen sind stärker betroffen (17\%) als im Osten (13\%).

Abstriche beim Entgelt werden in verschiedenen Formen realisiert. Unter den zum einen um Abstriche bei der Leistungshöhe der betrieblichen Altersversorgung und zum anderen um die Zugangsbeschränkung.

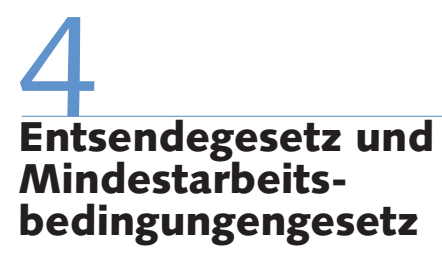

Die Große Koalition hatte sich im Sommer 2007 auf eine Ausweitung des Arbeitnehmerentsendegesetzes sowie auf eine Modernisierung des Mindestarbeitsbedingungengesetzes geeinigt, die Umsetzung dieses Beschlusses verlief allerdings sehr schleppend (Bispinck/WSI-Tarifarchiv 2008, 2009). Nach langen Kontroversen trat im April 2009 das neu gefasste Arbeitnehmer-Entsendegesetz in Kraft und die gesetzlichen Verfahren zur Festsetzung branchenbezogener Mindestlöhne konnten in den neu einbezogenen Branchen eingeleitet werden. Betroffen waren die Alten- und ambulante Krankenpflege, das Bewachungs- und Sicherheitsgewerbe, die Abfallwirtschaft, die berufliche Weiterbildung, die Wäschereidienstleistungen im Objektkundengeschäft sowie die Bergbauspezialdienste. Die entsprechenden Mindestlohntarifverträge lagen bereits vor. Für das Bewachungsgewerbe gab es einen Tarifvertrag der christlichen Gewerkschaft Öffentlicher Dienst und Dienstleistungen (GÖD), die übrigen Verträge waren von DGB-Gewerkschaften abgeschlossen worden.

Im Tarifausschuss beim Bundesarbeitsministerium wurde Ende August 2009 für zwei der Branchen eine Einigung über neue Mindestlohntarifverträge nach dem Entsendegesetz erzielt, nämlich für die Bergbauspezialdienste und die Wäschereidienstleistungen im Objektkundengeschäft. Für das Bewachungsgewerbe lehnte die Arbeitnehmerseite die Zustimmung ab, da hier mit $6 € z$ z. B. für das Saarland und Rheinland-Pfalz keine Existenz sichernden Löhne vereinbart werden sollten. Die Arbeitgeberseite blockierte Mindestlöhne für den Zweig Berufliche Weiterbildung. Für die Abfallwirtschaft signalisierte der Tarifausschuss Zustimmung, allerdings mussten hier noch letzte Details geklärt werden. Erst am 18.12. erfolgte dann die Rechtsverord-

2 Gefragt wurde nach dem Zeitraum von Anfang Juli 2008 bis Ende 2009. 
nung durch die neue Bundesarbeitsministerin von der Leyen, sodass die Mindestlöhne zu Beginn des Jahres 2010 in Kraft treten konnten (Übersicht 4).

Für den Bereich der Altenpflege wurde eine spezielle Kommission eingerichtet, die den Mindestlohn für diese Branche festlegen soll. Neben den Vertretern von Arbeitgebern und Gewerkschaften gehören dem Gremium auch Vertreter der Arbeitsrechtlichen Kommissionen der Wohlfahrtsverbände an.

Das neue Mindestarbeitsbedingungengesetz (MiArbG) ist ebenfalls seit April 2009 in Kraft. Der darin vorgesehene Hauptausschuss soll künftig prüfen, ob es in einem Wirtschaftszweig soziale Verwerfungen gibt und Mindestarbeitsentgelte festgesetzt, geändert oder aufgehoben werden sollen. Über die konkrete Höhe der Lohnuntergrenzen beschließen dann branchenspezifische Fachausschüsse. Die Bundesregierung kann schließlich den empfohlenen Mindestlohn für die jeweilige Branche per Rechtsverordnung branchenweit festlegen. Vorsitzender des Hauptausschusses ist der frühere SPD-Politiker Klaus von Dohnanyi. Weitere Mitglieder sind als Vertreter der Wissenschaft der Vorsitzende des Sachverständigenrates zur Begutachtung der gesamtwirtschaftlichen Entwicklung, Wolfgang Franz, und die Präsidentin des Wissenschaftszentrums Berlin für Sozialforschung, Jutta Allmendinger. Der DGB ist mit seinem Vorsitzenden Michael Sommer sowie Otto Ernst Kempen, Professor für Arbeitsrecht, vertreten. Die Arbeitgeberseite wird von BDA-Präsident Dieter Hundt und Otto Kenzler, dem Präsidenten des Zentralverbandes des Deutschen Handwerks, vertreten. Am 15.9. trat der Ausschuss zu seiner ersten Sitzung zusammen. Konkrete Fälle wurden bis zum Jahresende nicht bearbeitet.

Die schwarz-gelbe Regierungskoalition hat in ihrem Koalitionsvertrag vereinbart, eine gesetzliche Regelung zu sittenwidrigen Löhnen vorzunehmen. Damit soll klargestellt werden, was nach der Rechtsprechung ohnehin rechtswidrig ist. Nach Auffassung der Gewerkschaften ist damit in Bezug auf die Eingrenzung des Niedriglohnsektors konkret nichts gewonnen. Wenn nach einer solchen gesetzlichen Klarstellung auch tarif- oder ortsübliche Löhne um bis zu einem Drittel unterschritten werden dürfen, ohne dass dies als sittenwidrig gilt, bleiben künftig weiterhin Stundenlöhne von 3, 4 oder $5 €$ zulässig (WSI-Tarifarchiv 2009c).

\begin{tabular}{|c|c|c|c|}
\hline \multicolumn{4}{|c|}{$\begin{array}{l}\text { Übersicht 4: Tarifliche Mindestlöhne nach dem AEntG für } 2009 \\
\text { neu aufgenommene Branchen }\end{array}$} \\
\hline Branche & Beschäftigte & \multicolumn{2}{|c|}{ Mindestlohn/€ ab } \\
\hline Abfallwirtschaft & $\begin{array}{l}130.000 \\
\text { Mindestlohn }\end{array}$ & \multicolumn{2}{|c|}{$\begin{array}{l}01 / 2010 \\
8,02\end{array}$} \\
\hline \multirow[t]{2}{*}{ Bergbauspezialarbeiten } & $\begin{array}{l}2.500 \\
\text { Mindestlohn I }\end{array}$ & \multicolumn{2}{|l|}{$\begin{array}{c}01 / 2010 \\
11,17\end{array}$} \\
\hline & $\begin{array}{l}\text { Mindestlohn II } \\
\text { (Hauer/Facharbeiter) }\end{array}$ & \multicolumn{2}{|l|}{12,41} \\
\hline $\begin{array}{l}\text { Wäschereidienstleistungen im } \\
\text { Objektkundengeschäft }\end{array}$ & 35.000 & $01 / 2010$ & $04 / 2010$ \\
\hline West & Mindestlohn & 7,51 & 7,65 \\
\hline Ost inkl. Berlin & Mindestlohn & 6,36 & 6,50 \\
\hline \multicolumn{2}{|c|}{$\begin{array}{l}\text { Beschäftigtenzahlen: WSI-Tarifarchiv, BMA nach Angaben der Tarifparteien. } \\
\text { Quelle: WSI-Tarifarchiv, Stand: Januar } 2010 .\end{array}$} & \multicolumn{2}{|c|}{ WSI MITTEILUNGEN } \\
\hline
\end{tabular}

\section{5 \\ Ausblick auf die Tarif-
entwicklung 2010}

Die Tarifrunde 2010 bewegt sich zwischen zwei großen Themen: Beschäftigungssicherung und Einkommenserhöhung. Dabei werden die Akzente von den Gewerkschaften ganz unterschiedlich gesetzt: Im öffentlichen Dienst legen ver.di und die dbb-tarifunion den Schwerpunkt auf Entgelterhöhungen. Zum Jahresende 2009 sind die Entgelttarifverträge für die Beschäftigten bei Bund und Gemeinden ausgelaufen. Ver.di fordert ein Tarifvolumen von $5 \%$. Darin enthalten sind eine „spürbare“ Entgelterhöhung, strukturelle Entgeltverbesserungen, die Fortführung der Altersteilzeit sowie spezifische Forderungen für einzelne Bereiche wie Krankenhäuser, Pflegeeinrichtungen und Versorgungsbetriebe. In der Metallindustrie, wo die Tarifverträge Ende April auslaufen, hat der IG MetallVorsitzende Huber bereits deutlich ge- macht, dass das alles überragende Ziel die (tarifliche) Sicherung von Beschäftigung sei, während für Entgelterhöhungen „die Luft sehr dünn" sei. Zur Beschäftigungssicherung ist vor allem an eine Ausweitung der Beschäftigungssicherungstarifverträge gedacht, sodass eine Absenkung der Wochenarbeitszeit auf bis zu 25 Stunden mit teilweisem Lohnausgleich möglich wird. Für die chemische Industrie hat die IG BCE in einer Forderungsempfehlung neben einer „angemessenen“ Entgelterhöhung ebenfalls Maßnahmen zur Beschäftigungssicherung gefordert. In anderen Branchen und Tarifbereichen fordern die Gewerkschaften bislang Lohnund Gehaltserhöhungen zwischen 3,5\% und $5 \%$.

In einer Reihe von Tarifbereichen wurden bereits Tariferhöhungen für das Jahr 2010 vereinbart. Die im kommenden Jahr in Kraft tretenden Steigerungsraten bewegen sich zwischen 1,2\% und 6,2\% (Übersicht 5). Damit zeichnet sich für das Jahr 2010 eine (deutlich) niedrigere Steigerung der Tarifverdienste ab als im Jahr zuvor.

\begin{tabular}{|c|c|c|c|}
\hline \multicolumn{4}{|l|}{ Übersicht 5: Tariferhöhungen im Jahr 20101) } \\
\hline Branche & ab Monat & $\%$ & Laufzeit bis \\
\hline Bauhauptgewerbe West/Ost & 04 & $2,3 / 2,6$ & $03 / 2011$ \\
\hline Deutsche Bahn AG & 01 & 2,0 & $07 / 2010$ \\
\hline Deutsche Telekom AG & 01 & 2,5 & $12 / 2010$ \\
\hline Druckindustrie & 04 & 2,0 & 03/2011 \\
\hline Einzelhandel Nordrhein-Westfalen & 09 & 1,5 & $04 / 2011$ \\
\hline Eisen- und Stahlindustrie Niedersachsen, Bremen, NRW & 01 & 2,0 & 08/2010 \\
\hline Gebäudereinigerhandwerk West inkl. Berlin/Ost & 01 & $3,1 / 3,8-6,22)$ & $12 / 2011$ \\
\hline Groß- und Außenhandel Sachsen-Anhalt & 06 & 1,5 & $04 / 2011$ \\
\hline Holz und Kunststoff verarbeitende Industrie Niedersachsen & 11 & 1,7 & 04/2011 \\
\hline Öffentlicher Dienst Länder & 03 & 1,2 & $12 / 2010$ \\
\hline RWE & 01 & 2,2 & $10 / 2010$ \\
\hline Süßwarenindustrie Baden-Württemberg & 07 & 1,9 & 06/2011 \\
\hline Textil- und Bekleidungsindustrie West & 01 & $1,5^{3)}$ & $02 / 2011$ \\
\hline Versicherungsgewerbe & 04 & 2,5 & $03 / 2011$ \\
\hline \multicolumn{4}{|l|}{$\begin{array}{l}\text { 1) Vereinbart in Tarifabschlüssen der Tarifrunde } 2009 . \\
\text { 2) regional unterschiedlich. } \\
\text { 3) plus } 40 € \text { Sockelbetrag. }\end{array}$} \\
\hline Quelle: WSI-Tarifarchiv 2010. & & IVIII & EILUNGEN \\
\hline
\end{tabular}




\section{LITERATUR}

Bispinck, R. (Hrsg.) (2008): Verteilungskämpfe und Modernisierung. Aktuelle Entwicklungen in der Tarifpolitik, Hamburg

Bispinck, R./Schulten T. (2008): Aktuelle Mindestlohndebatte: Branchenlösungen oder gesetzlicher Mindestlohn?, in: WSI-Mitteilungen 3 ,

S. 151-158

Bispinck, R./WSI-Tarifarchiv (2008): Tarifpolitischer Jahresbericht 2007: Gespaltene Tarifentwicklung und verschärfte Gewerkschaftskonkurrenz, in: WSI-Mitteilungen 2, S. 77-84

Bispinck, R./WSI-Tarifarchiv (2009): Tarifpolitischer Jahresbericht 2008 : Tarifpolitik in der Finanzmarktkrise, Düsseldorf, 57 Seiten

Bogedan, C./Brehmer, W./Herzog-Stein, A. (2009): Betriebliche Beschäftigungssicherung in der Krise - Eine Kurzauswertung der WSI-Betriebsrätebefragung 2009, WSI-Aktuell 01/Dezember

Bundesinstitut für Berufsbildung (BiBB) (2009): Tarifliche Ausbildungsvergütungen 2009 - Stärkster Anstieg seit 14 Jahren, Pressemitteilung $1 / 2010$ vom 5.1.2010

Bundesvereinigung der Deutschen Arbeitgeberverbände (BDA) (2009) Geschäftsbericht 2009, Berlin

Ellguth, P./Kohaut, S. (2008): Tarifbindung und betriebliche Interessenvertretung: Aktuelle Ergebnisse aus dem IAB-Betriebspanel 2007, in: WSI-Mitteilungen 9, S. 515-522

Gewerkschaft Erziehung und Wissenschaft (GEW) (2007): „Erzieherinnen verdienen mehr. " Expertise und Portraits zur Gleichwertigkeit der Tätigkeit von Erzieherinnen und Erziehern mit anderen Fachschulberufen. Erstellt im Auftrag der Max-Traeger-Stiftung der GEW von Dr. Andrea Jochmann-Döll unter Mitarbeit von Dr. Karin Tondorf. Portraits von Gesine Kulcke, Frankfurt am Main
Gewerkschaft Erziehung und Wissenschaft (GEW) (2009): Arbeitsqualität aus Sicht von Erzieherinnen und Erziehern. Ergebnisse aus der Erhebung zum DGB-Index Gute Arbeit, erstellt von Tatjana Fuchs und Falko Trischler

Hundt, D. (2009): Mit Mut und Zuversicht aus der Krise - Rede auf dem Deutschen Arbeitgebertag 2009, am 24.11. in Berlin

IG Metall (2009): Keine Entlassungen in der Krise - industrielle Wertschöpfung sichern. Aktuelle Handlungslinien der Krisenbewältigung, Frankfurt/Main, November Institut für Makroökonomie und Konjunkturforschung (IMK) (2009): Deutsche Arbeitskosten im europäischen Vergleich: Nur geringer Anstieg, Auswertung der aktuellen Eurostat-Statistik für 2008, IMK-Report 44, November

Sommer, M. (2010): Tarifautonomie und Tarifpolitik - für eine Stabilisierung des Tarifsystems, in: Bispinck, R./Schulten, T. (Hrsg.): Zukunft der Tarifautonomie. 60 Jahre Tarifvertragsgesetz: Bilanz und Ausblick, Hamburg, S. 13-19

Statistisches Bundesamt (2010): Volkswirtschaftliche Gesamtrechnung 2009, vorläufige Ergebnisse, Wiesbaden

WSI-Tarifarchiv (2009a): Tarifliche Regelungen zur Kurzarbeit. Ein aktueller Überblick über 18 Wirtschaftszweige und Tarifbereiche, Elemente qualitativer Tarifpolitik 66, Düsseldorf, Februar WSI-Tarifarchiv (2009b): Tarifliche Regelungen zur befristeten Arbeitszeitverkürzung. Eine Untersuchung von Tarifverträgen in 26 Wirtschaftszweigen und Tarifbereichen, Elemente qualitativer Tarifpolitik 67, Düsseldorf, Juni

WSI-Tarifarchiv (2009c): Analyse des WSI-Tarifarchivs: Gesetzliche Grenze für sittenwidrige Löhne schafft keinen angemessenen Mindestlohn, Pressemeldung vom 21.10.2009 